Revisão / Review

\title{
Efeitos do exercício e do treinamento físico na hemostasia
}

\section{Exercise and training effects on blood haemostasis}

Jerri L. Ribeiro ${ }^{l}$

Alvaro R. Oliveira ${ }^{2}$
O estado hipercoagulante pós-exercício tem sido bem demonstrado, pois aumentos na atividade coagulante são freqüentemente registrados após diversos protocolos de exercício. Um dos efeitos agudos do exercício mais consistente é o aumento da atividade coagulante refletido pelo aumento da atividade do FVIII, dos marcadores TAT e F1+2. Esse aumento é intensidade dependente. Quanto aos efeitos crônicos, a atividade coagulante de repouso não se modifica com o treinamento. Os níveis de FVIII pósexercício, entretanto, são maiores em sedentários do que em treinados e os níveis de repouso de pacientes pós-infarto diminuem com o treinamento. A hiperfibrinólise provocada pelo exercício também está comprovada e, assim como a atividade coagulante, parece depender da intensidade. Foram registrados aumentos dos niveis de t-PA, u-PA, plasminogênio e diminuição dos níveis de PAI-1 após o exercício. Os níveis de repouso de t-PA de pessoas inativas é maior que em ativos e atletas. Além disso, pacientes pós-infarto apresentaram diminuição significativa dos níveis de PAI-1 após periodo de reabilitação física. Os dados mais controversos estão relacionados aos efeitos crônicos dos níveis de t-PA. Enquanto estudos demonstram níveis maiores de t-PA em treinados comparados a sedentários, outros mostram o contrário. Resultados mais consistentes acerca dos efeitos do exercício no processo hemostático, incluindo atividades coagulante e fibrinolítica, ainda são necessários. Existe ainda carência quanto aos efeitos crônicos do exercício, tanto em relação aos beneficios na saúde quanto à influência na performance de atletas. Rev. bras. hematol. hemoter. 2005; 27(3):213-220.

Palavras-chave: Coagulação; fibrinólise; hemostasia; exercício; treinamento.

\section{Introdução}

O estudo dos efeitos do exercício no mecanismo da coagulação sangüínea tornou-se importante devido à necessidade de se entender suas relações com a patogênese de distúrbios vasculares, já que o exercício parece modificar sua atividade e progressão. Essa preocupação já foi demonstrada no início do século passado através de estudos que investigavam o estado hipercoagulável pós-exercício. ${ }^{1}$ Exis- tem evidências de que o exercício altera as três cascatas proteolíticas envolvidas na hemostasia: coagulação, fibrinólise e sistema complementar. ${ }^{2}$ A partir disso, diversas investigações têm sido feitas, porém muitos resultados são freqüentemente conflitantes devido às diferenças nos protocolos, estado de treinamento e de saúde dos indivíduos testados e métodos de análise.

Muitas investigações têm utilizado uma variedade de protocolos de exercício que estudam as alterações na coa-

\footnotetext{
${ }^{1}$ Doutorando em Ciências do Movimento Humano - EsEF/UFRGS.

${ }^{2}$ Professor Adjunto de Fisiologia do Exercício - EsEF/UFRGS.

Laboratório de Pesquisa do Exercício (LAPEX) - Escola de Educação Física - Universidade Federal do Rio Grande do Sul (UFRGS).
}

Correspondência para: Jerri L. Ribeiro

Programa de Pós-Graduação

Rua Felizardo, 750 - Jd. Botânico

90690-200 - Porto Alegre-RS

Telefone: (51) 33165861/30285235 - Fax: (51)33165811

E-mail: jerriribeiro@yahoo.com.br 
gulação e sistema fibrinolítico que incluem corrida até a exaustão, caminhada, ciclismo e corrida em esteira, entre outros. Muitos não controlaram a intensidade e duração, o que pode ser uma causa para a variabilidade nas observações. Em alguns estudos em que os indivíduos se exercitaram em um grau similar de intensidade relativa de esforço, como indicado pelo percentual do consumo máximo de oxigênio ( $\mathrm{VO}_{2}$ máx), ou percentual da freqüência cardíaca máxima, os resultados foram mais consistentes. A maioria das investigações registrou aumentos induzidos pelo exercício de diversos fatores de coagulação, principalmente o fator VIII; entretanto, alguns não têm dado atenção à correção pela hemoconcentração. Andrew et $\mathrm{al}^{3}$ não encontraram alterações significativas para os fatores XII, V, II, ou fibrinogênio após a correção, dando suporte à conclusão de Huisveld et $\mathrm{al}^{4} \mathrm{de}$ que, excluindo o complexo fator VIII, a concentração de outros fatores de coagulação não se altera durante o exercício. O primeiro estudo ainda encontrou forte relação do aumento da atividade do complexo fator VIII à intensidade do exercício, pois intensidades máximas de exercício de curta ou longa duração induziram fortes elevações de todos os componentes do fator VIII e das concentrações de lactato (La) e hidrogênios livres $\left(\mathrm{H}^{+}\right)$. Alguns autores sugerem que o aumento no fator VIII é mediado via receptores $\alpha$-adrenérgicos e que a agregação plaquetária é induzida por uma atividade sinérgica entre a concentração de catecolaminas e o estresse de cisalhamento (shear stress). O mecanismo pelo qual este aumento é induzido ainda não está bem esclarecido, por isso mais investigações são necessárias.

\section{Coagulação e exercício}

A ativação da coagulação resulta na formação de trombina, no aumento dos níveis de fibrinopeptídeo A, de fatores coagulantes ativos e de marcadores como o fragmento ativador de protrombina $1+2(\mathrm{~F} 1+2)$, resultando num estado hipercoagulante pós-exercício com encurtamento no tempo de coagulação sangüínea e diminuição no tempo de ativação parcial da tromboplastina (APTT).

Diversos estudos demonstraram que o exercício estimula o aumento dos níveis do complexo VIII, que é o principal responsável pelo incremento no estado funcional do sistema coagulante, como evidenciado pelo encurtamento pósexercício do APTT. ${ }^{2} \mathrm{O}$ complexo VIII é composto por dois polipeptídeos distintos, o fator VIII:C, que manifesta a atividade pró-coagulante (deficiente em pacientes com hemofilia clássica), e fator von Willebrand, VIII:vWF (proteína de adesão plaquetária deficiente em pacientes com doença de von Willebrand). A concentração molar do fator von Willebrand é aproximadamente 100 vezes a do fator VIII:C. Virtualmente, todo o fator VIII:C existe no plasma em um complexo não covalente com o fator VIII:vWF. Estes dois fatores, que têm controles genéticos diferentes, mas circulam juntos no plasma, podem ser dissociados por métodos físicos como em soluções altamente salinizadas, cromatografia de troca iônica ou por filtração gel na presença de detergentes.

O fator VIII:vWF foi identificado em megacariócitos, plaquetas e células endoteliais; quanto ao fator VIII:C ,existem indicações de que seja sintetizado pelo baço e fígado. Três métodos de análise para os dois fatores do complexo VIII são rotineiramente empregados. Análises funcionais existem para a atividade pró-coagulante (FVIII:C) e a atividade do co-fator ristocetina para o fator von Willebrand. $O$ fator von Willebrand também é quantificado imunoquimicamente através do antígeno relacionado ao fator VIII (FVIII:Rag). Todos os três determinantes parecem aumentar após o exercício.

Muitos estudos demonstram que aumentos equivalentes ocorrem entre FVIII:C e FVIII:Rag. ${ }^{6-9}$ Existem, entretanto, registros de aumentos desproporcionais do FVIII:C sobre FVIII:vWF e FVIII:Rag. ${ }^{10-12}$ As diferenças nos protocolos de exercício não explicam facilmente as diferenças nos resultados. Diferenças nos métodos laboratoriais, entretanto, podem ser parcialmente responsáveis. A atividade do FVIII:C no plasma de um indivíduo é determinada por uma expansão no APTT, no qual o plasma diluído é adicionado a um substrato plasmático deficiente em FVIII:C e a concentração determinada pela comparação do tempo de formação do coágulo com uma curva padrão. Brown et a ${ }^{13}$ demonstraram que o APTT de um estágio no qual o plasma do indivíduo e o substrato são inicialmente misturados resulta em níveis mais altos de FVIII:C do que o método de dois estágios, onde o plasma é primeiramente ativado e então adicionado ao substrato plasmático. Todos os estudos que demonstraram aumentos desproporcionais no FVIII:C sobre FVIII:RAg utilizaram o método de um estágio, e todos, exceto um, que demonstraram um aumento proporcional, 7 usaram a técnica de dois estágios. Não existe uma razão clara para esta súbita diferença nas técnicas produzir resultados distintos na atividade do FVIII:C. Kopitsky et a ${ }^{14}$ mostraram aumento desproporcional na atividade do FVIII:C com exercício usando a técnica de um ou dois estágios e observaram uma relação positiva entre o aumento da atividade do FVIII:C e a ativação de trombina. Seus estudos evidenciam que um aumento desproporcional no FVIII:C após o exercício pode ser devido ao aumento na ativação in vivo do FVIII:C pela trombina. A possibilidade de quantificar os níveis plasmáticos do FVIII:C antígeno (em oposição à atividade funcional) pelo uso de anticorpos monoclonais pode esclarecer os problemas relacionados às elevações induzidas pelo exercício do FVIII:C. ${ }^{5}$

O estímulo responsável pelos aumentos induzidos pelo exercício no FVIII pode ser mediado por receptores $\beta$ adrenérgicos, pois betabloqueadores parecem reduzir ou, até mesmo, eliminar estes efeitos. ${ }^{8,15,16}$ Baseados nas evidências de que os efeitos adrenoceptores beta 1 e beta 2 podem ser mediados pelo óxido nítrico (NO), Jilma et a ${ }^{15}$ mostraram que o aumento do fator von Willebrand e FVIII:C no plasma durante o exercício é causado por um mecanismo NO dependente. Em seu estudo, mostraram uma atenuação nos aumen- 
Tabela 1

Aumentos no fator von Willebrand (vWF-Ag) e fator VIII:C durante exercício exaustivo em bicicleta com infusão de placebo ou do inibidor da NO-sintase L-NMMA (N-monometil-L-arginina)

\begin{tabular}{lcc}
\hline & Placebo & L-NMMA \\
\hline VWF-Ag & $61 \%$ & $25 \%$ \\
FVIII:C & $44 \%$ & $12 \%$ \\
\hline
\end{tabular}

Modificado de Jilma et al (1997)

tos induzidos pelo exercício após bloqueio parcial da NOsintase (Tabela 1), propondo que os processos físiológicos induzidos pela estimulação dos adrenoceptores beta 2 sistêmicos podem ser parcialmente mediados pelo NO. Ikarugi et $\mathrm{al}^{17}$ estudaram os efeitos agudos do exercício aeróbio e das catecolaminas no estado trombótico através de um método in vitro de formação do plug hemostático (hemostatometria). Homens voluntários saudáveis praticaram um exercício aeróbio ( $60 \%$ do $\mathrm{VO}_{2}$ máx) por vinte minutos, e os efeitos na reatividade plaquetária e coagulação foram medidos realizando-se a hemostatometria antes e imediatamente após o exercício. Os principais achados do estudo foram que a elevação da noradrenalina circulante a níveis obtidos durante o exercício causa hiperreatividade plaquetária e aumento na coagulação mediada pelas plaquetas. Além disso, Wagner et al ${ }^{18}$ encontraram resultados que indicam atividade sinergística entre a adrenalina e o estresse de cisalhamento (shear stress) na indução da agregação plaquetária. Esta resposta sinérgica requer complexos funcionais Gp IIb/IIIa (glicoproteínas plaquetárias), mas é parcialmente independente da interação vWF-GpIb.

\section{Efeitos agudos do exercício na coagulação}

Os efeitos do exercício na atividade coagulante do FVIII foram estudados através de diferentes protocolos com diversas intensidades e durações. Apesar desta variedade, todos relatam aumentos significativos desta atividade., ${ }^{2,19-23}$ Esses efeitos também foram observados após exercícios de força.

Neste estudo, ${ }^{24}$ o grupo que realizou os exercícios com maior volume (8-12 repetições) e menor intensidade (70\%-80\% de 1 repetição máxima, 1RM) demonstrou maior resposta no percentual da atividade do FVIII, após estes exercícios, comparado com o grupo que realizou com menor volume (1-5 repetições) e maior intensidade ( $90 \%-100 \%$ de $1 \mathrm{RM})$ e com o grupo controle.
O aumento da atividade do FVIII está associado à intensidade do exercício, e este aumento persiste durante a recuperação. Andrew et al ${ }^{3}$ estudaram três protocolos diferentes de exercício (exercício progressivo até a exaustão, exercício em steady state e exercício máximo de 30 segundos) e observaram que os componentes do complexo VIII aumentaram abruptamente (75\%-250\%) somente nas cargas mais altas e eram refletidas pelo aumento das concentrações plasmáticas de La e $\mathrm{H}+$ (Figura 1). Davis et $\mathrm{al}^{25}$ mostraram que o aumento da atividade do FVIII não aconteceu até que o consumo de oxigênio alcançasse $95 \%$ a $100 \%$ do máximo em um teste de esteira. Os efeitos de uma intensidade máxima de exercício persistem por, pelo menos, uma hora. Além disso, a intensidade do exercício influenciou também as concentrações do fator von Willebrand (vWF), já que Salton et $\mathrm{a}^{26}$ mostraram aumentos significativos nas respostas das concentrações plasmáticas do vWF de indivíduos jovens saudáveis em três intensidades diferentes de exercício em cicloergômetro.

Quando colocadas em um tubo de vidro, amostras de sangue pós-exercício tendem a coagular mais rápido do que amostras sangüíneas de repouso (whole blood clotting time - WBCLT). Essa tendência à hipercoagubilidade é também refletida pelo decréscimo (7\%-38\%) no APTT observado após exercícios máximos, ${ }^{6,27-30}$ inclusive isométricos. ${ }^{31}$

Os resultados sobre o tempo de protrombina $(\mathrm{PT})$ e o tempo de trombina (TT) em resposta ao exercício são controversos. Pesquisas demonstraram significativo encurtamento ${ }^{32}$ associado a uma diferença não significativa no $\mathrm{PT}^{7,22,33-36}$ após o exercício. Além disso, El-Sayed et al ${ }^{22}$ demonstraram que o exercício provocou diminuição significativa no TT. As alterações no APTT e PT persistem de 1 a 24 horas pósexercício. $^{21,33}$

Os efeitos agudos do exercício na concentração de fibrinogênio plasmático também são muito controversos, pois diversos estudos observaram diferenças não-signifi-

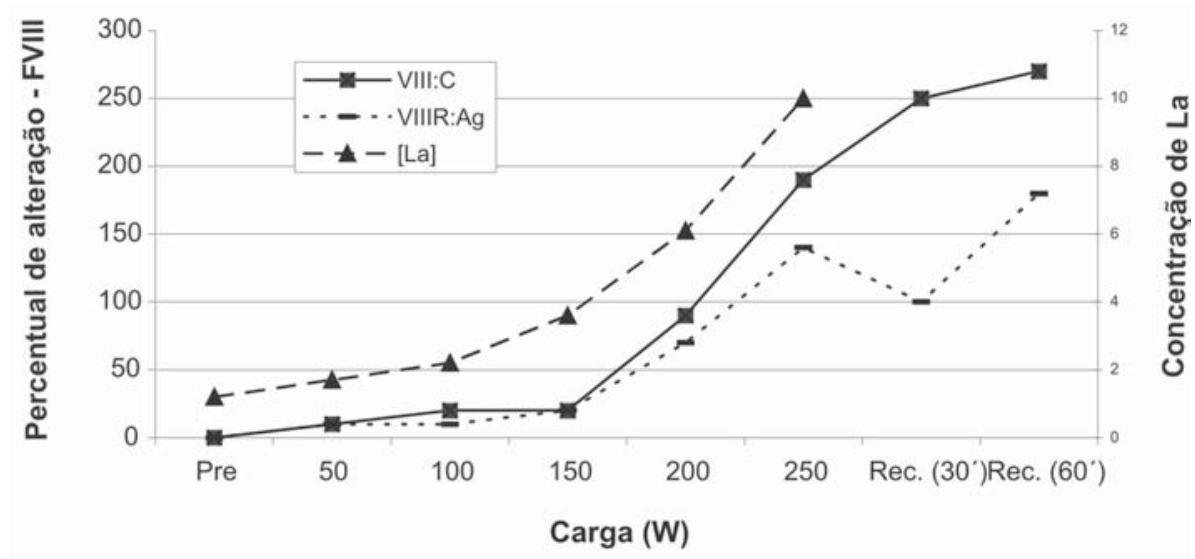

Figura 1. Lactato plasmático e fator VIII durante exercício progressivo até a exaustão e 30 e 60 minutos após. Modificado de Andrew et al (1986) 
cativas $^{22,37,38}$ enquanto outros registraram aumentos significativos ${ }^{21,39,40}$ ou diminuição significativa. ${ }^{41,42}$ El-Sayed et $\mathrm{al}^{43}$ sugerem que estes resultados conflitantes aparecem devido às diferenças nos protocolos, nível de aptidão física e saúde dos indivíduos testados e aos métodos de análise utilizados pelos estudos. Outros marcadores da ativação da coagulação sangüínea no exercício são o complexo trombinaantitrombina (TAT) e os fragmentos de protrombina $1+2$ $(\mathrm{F} 1+2)$ e aumentos significativos em ambos, TAT e F1+2, foram registrados pós-exercício. ${ }^{2,37,41,44,45} \mathrm{~A}$ formação de fibrinopeptídeo A (FPA), um marcador da hipercoagubilidade, é controversa; existem registros de aumentos significativos ${ }^{2,41}$ e não significativos. ${ }^{37,44}$

\section{Efeitos crônicos do exercício na coagulação}

Os efeitos do treinamento na atividade coagulante não são conclusivos pois existem poucos estudos nesta área e a variabilidade nos protocolos utilizados é grande. Alguns estudos não encontraram alterações e outros, um pequeno aumento no potencial coagulante após o treinamento. ${ }^{5,45-48}$ Já os trabalhos que verificaram os níveis de repouso do FVIII e do seu antígeno não encontraram diferença significativa, antes e após o período de treinamento, tanto em sedentári$\mathrm{os}^{48-50}$ como em atletas de endurance. ${ }^{51}$

A resposta aguda ao exercício, após 12 semanas de treinamento aeróbio, parece não se modificar em indivíduos saudáveis, porém, quando testada em indivíduos após infarto do miocárdio, a atividade do FVIII e do antígeno diminui após quatro semanas de treinamento. ${ }^{39}$ Van den Burg et al ${ }^{19}$ não observaram alterações nos níveis plasmáticos de repouso das variáveis hemostáticas em jovens e em indivíduos idosos, após o mesmo período de treinamento, apesar do aumento da aptidão cardiorrespiratória $\left(\mathrm{VO}_{2} \mathrm{máx}\right)$. É importante notar que não houve alterações na massa corporal e no percentual de gordura, dando suporte às observações de que as alterações na composição corporal per se estão associadas às alterações na coagulação e atividade fibrinolítica. ${ }^{48,52}$ Este mesmo estudo ainda encontrou uma hipercoagubilidade induzida pelo exercício agudo mais elevada e níveis mais altos durante a recuperação. Kvernmo et $\mathrm{al}^{45}$ identificaram ativação mais pronunciada do sistema coagulante entre os controles $\left(\mathrm{VO}_{2}\right.$ máx. $\left.=52,6 \mathrm{ml} \cdot \mathrm{kg}^{-1} \cdot \mathrm{min}^{-1}\right)$ comparados a atletas $\left(\mathrm{VO}_{2}\right.$ máx. $\left.=68,4 \mathrm{ml} \cdot \mathrm{kg}^{-1} \cdot \mathrm{min}^{-1}\right)$ quando avaliados após exercício a $80 \%$ do $\mathrm{VO}_{2}$ máx., e estes resultados (Figura 2) são consistentes com outros estudos. ${ }^{30,53-55}$

Estudos que verificaram outras variáveis do sistema coagulante como o PT, APTT e o TT não encontraram diferenças significativas em repouso e pós-exercício ${ }^{32,51,56}$ Quanto aos níveis plasmáticos de fibrinogênio, estudos epidemiológicos mostram uma associação favorável com o treinamento físico. ${ }^{22,57}$ Algumas pesquisas relatam a redução no fibrinogênio em pacientes ${ }^{58} \mathrm{e} e \mathrm{~m}$ homens idosos, mas não em jovens. Em contraste, foi encontrado um aumento na concentração de fibrinogênio em homens idosos após treina-

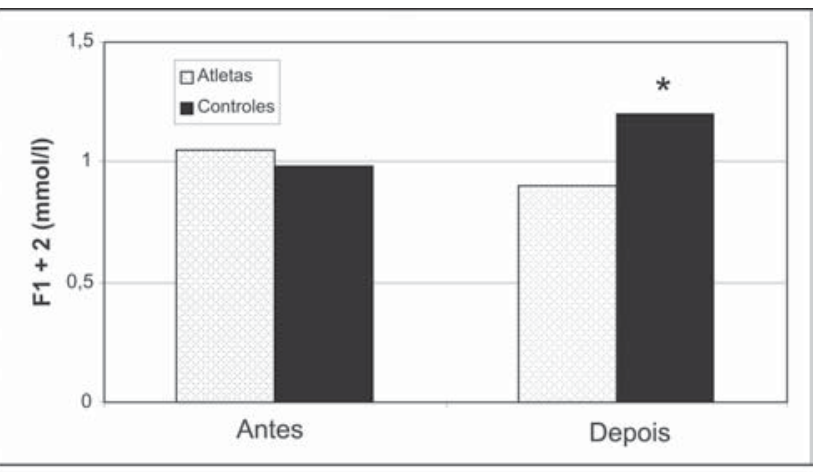

Figura 2. Níveis plasmáticos do fragmento de protrombina $1+2(F 1+2)$ antes e após exercício padronizado em atletas $(n=8)$ e controles $(n=8) .{ }^{*} p<0,05$ dentro do grupo. Modificado de Kvernmo et al (1992)

mento intenso $\mathrm{o}^{59} \mathrm{e}$, em mulheres idosas, tais efeitos parecem ser negligíveis. ${ }^{52}$

\section{Efeitos do exercício na função plaquetária}

A trombocitose (aumento na contagem de plaquetas) foi registrada após exercícios extenuantes aumentando de $18 \%$ a $80 \%$. Este aumento acontece em resposta a diversos agentes agregatórios como o ADP, colágeno e adrenalina. Além disso, houve aumento na $\beta$-tromboglobulina ( $\beta$ TG) e no fator plaquetário 4 (PF4) plasmáticos, indicando aumento na agregação plaquetária. Os aumentos na $\beta$ TG em resposta ao exercício permanecem dentro da faixa fisiológica e, por isso, a liberação plaquetária in vivo é considerada mínima. ${ }^{43}$

A ativação plaquetária induzida pelo exercício esteve relacionada ao metabolismo anaeróbio, pois ela é mais pronunciada em cargas de exercício acima do limiar anaeróbio. ${ }^{60-62}$ A participação das catecolaminas no aumento da agregação plaquetária ainda é questionável pois estudos que utilizaram $\beta$-bloqueadores encontraram inibição desta atividade enquanto outros não registraram o mesmo efeito. Quando utilizados bloqueadores dos canais de cálcio, uma atenuação importante da agregação plaquetária foi encontrada. $\mathrm{O}$ mecanismo da redução desta agregação após exercício, encontrada em estudos com hipertensos e sujeitos saudáveis, não está bem esclarecido, mas parece estar ligado ao aumento da liberação de prostaciclina (prostanóide antiagregatório) ou t-PA, que desagrega plaquetas. Com relação aos efeitos do treinamento, os resultados ainda são controversos; entretanto, alguns estudos observaram uma diminuição na adesividade e agregação plaquetárias no repouso e pós-exercício, após treinamento de endurance. ${ }^{43}$

\section{Fibrinólise e exercício}

$\mathrm{O}$ aumento da atividade fibrinolítica, com aumento na formação de plasmina, resulta na liberação do ativador do plasminogênio tecidual (t-PA) e no ativador do plasminogênio uroquinase (u-PA). Existe uma variação diurna dos níveis de atividade fibrinolítica de indivíduos em repouso com os valores mais baixos pela manhã, e os mais altos (4 vezes o basal) 
no início da tarde, período em que as respostas mais intensas ao exercício foram notadas. Existe uma grande variação entre indivíduos, mas, para cada indivíduo, os níveis basais e respostas tendem a ser similares dia a dia. O exercício intenso induz o aumento da atividade fibrinolítica atribuído ao aumento dos níveis de t-PA. Esta enzima é sintetizada e estocada no endotélio vascular e liberada em resposta a diversos estímulos que incluem medo, estresse cirúrgico, oclusão venosa, hipoglicemia, desmopressina e diversos medicamentos. O aumento do fluxo sangüíneo venoso pode representar um mecanismo comum a alguns destes estímulos. ${ }^{5}$ Há evidências de aumento plasmático dos níveis de u-PA pós-exercício. Deve ser observado, no entanto, que os níveis de pico de u-PA e t-PA não coincidem, em tempo ou magnitude, em resposta ao exercício máximo. Isto pode significar que mecanismos independentes regulam os aumentos induzidos pelo exercício nos níveis de u-PA e t-PA. ${ }^{43}$

Adicionalmente à secreção de t-PA endotelial, o exercício pode induzir alterações no seu substrato, o plasminogênio. Quantidade aumentada de plasminogênio modificado foi registrada em plasma pós-exercício. Esta forma de plasminogênio tem grande afinidade por fibrina e é mais facilmente ativada pelo ativador do plasminogênio. ${ }^{5}$ Estudos indicam a existência de poor responders (indivíduos que demonstram uma resposta fibrinolítica diminuída ao exercício) entre indivíduos saudáveis, apesar de mais freqüente entre pacientes. Isto demonstra que a habilidade de responder adequadamente ao exercício representa a capacidade do potencial fibrinolítico. Conseqüentemente, poor responders estão provavelmente sob um risco maior de doença vascular aterosclerótica quando submetidos ao exercício. ${ }^{43}$

Efeitos agudos do exercício na atividade fibrinolítica

Existem registros de aumentos de $75 \%$ a $250 \%$ da atividade fibrinolítica que ocorrem em intensidades onde a freqüência cardíaca atinge $50 \%$ ou mais do seu máximo ${ }^{3}$ e os maiores aumentos ocorrem entre $70 \%$ e $90 \%$ da carga máxima de trabalho. ${ }^{3,25}$ Alguns conflitos acontecem quanto ao retorno aos valores de repouso de t-PA e u-PA, pois estudos registram 45 a 60 minutos, após exercício intenso, ${ }^{27,32}$ duas horas após corrida de longa distância ${ }^{23}$ e 24 horas após maratona. ${ }^{42}$

Aumentos significativos também aparecem na atividade do plasminogênio tecidual e do seu antígeno em diferentes protocolos de exercício ${ }^{21,33,34,38,42,63-65}$ e estes aumentos são intensidade dependentes. ${ }^{34,66} \mathrm{~A}$ atividade do t-PA também aumenta em trabalhos de força dependendo do volume. ${ }^{24} \mathrm{Já}$ em exercício isométrico máximo, Röcker et al ${ }^{31}$ não encontraram alteração na atividade do t-PA, mas um leve aumento na concentração do t-PA antígeno. Diminuição significativa na atividade do PAI-1 (inibidor do ativador do plasminogênio) ocorre após exercícios aeróbios e anaeró$\operatorname{bios}^{22,63,65,66}$ exercícios em condições hipoxêmicas ${ }^{67}$ e exercícios de força. ${ }^{24}$ Recentemente, Womack et a ${ }^{68}$ encontraram aumento significativo (180\%) na atividade do t-PA e diminuição (40\%) na atividade do PAI-1 sem alterações nos antígenos em pacientes com doença arterial periférica. Os níveis da atividade do t-PA plasmático permaneceram elevados ( $80 \%$ ) até uma hora após o exercício e a diminuição da atividade do PAI-1 (49\%). Houve ainda uma correlação significativa $(\mathrm{p}<0,05)$ da diminuição no PAI-1 com o consumo de oxigênio $\left(\mathrm{VO}_{2}\right)$.

Outra maneira de verificar as alterações na atividade fibrinolítica é através dos marcadores da degradação do fibrinogênio e/ou fibrina ( $\mathrm{Fb} / \mathrm{FgDP})$. Aumentos significativos de $\mathrm{Fb} / \mathrm{FgDP}$ foram registrados após diferentes protoco$\operatorname{los}^{32,42}$ e relacionados com a intensidade e grau de treinamento dos indivíduos. ${ }^{63,69}$ Aumentos nas concentrações do Ddimer - outro marcador da hiperfibrinólise - ocorreram após exercício submáximo seguido de exercício curto máximo ${ }^{34} \mathrm{e}$ após exercícios de endurance. ${ }^{21,33,42,44}$

Efeitos crônicos do exercício na atividade fibrinolítica Os efeitos do exercício na fibrinólise são favoráveis mas, quanto ao treinamento, os resultados ainda são inconsistentes. Sujeitos inativos apresentaram maior atividade do t-PA e seu antígeno em repouso que pessoas fisicamente ativas ${ }^{63,66}$ e atletas. ${ }^{45}$ Além disso, pacientes após infarto do miocárdio, que possuem níveis mais altos de PAI-1 quando comparados a idosos, ${ }^{70}$ sofrem redução significativa ao serem submetidos a programas de reabilitação, ao contrário dos controles. ${ }^{39,71}$

A hiperfibrinólise após o exercício está relacionada ao grau de treinamento dos indivíduos (Ferguson et al, 1987), ${ }^{32}$ pois a liberação de t-PA parece ser maior e o complexo t-PA/ PAI menor em indivíduos treinados comparados a sedentários. ${ }^{63,66}$ Em contraponto, Kvernmo et a ${ }^{45}$ encontraram liberação mais baixa de t-PA, em repouso e após exercício, em atletas comparados aos controles (Figura 3). Essa adaptação na resposta fibrinolítica foi demonstrada nos níveis de PAI-1 após o exercício, onde os atletas possuíam valores mais baixos.

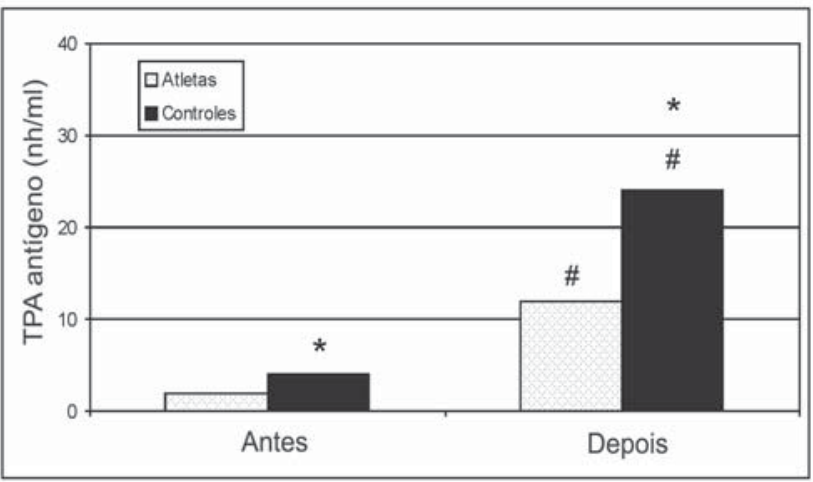

Figura 3. Concentração plasmática do ativador do plasminogênio tecidual (t-PA) antes e após exercício padrão em atletas $(n=8)$ e controles $(n=8) .{ }^{*} p<0,05$ entre os grupos e \# $p<0,005$ dentro dos grupos. Modificado de Kvernmo et al (1992) 
Altos níveis de PAI-1 estariam associados com um aumento no complexo t-PA/PAI, o que significa que o t-PA estaria sendo bloqueado na sua função de ativação do plasminogênio à plasmina. Os autores justificam a menor liberação do t-PA pelos atletas por uma maior resistência das células endoteliais como efeito do exercício regular. Apesar de altos níveis de t-PA favorecerem a dissolução de fibrina, elevados níveis de t-PA antígeno estiveram fortemente associados com riscos de futuro infarto do miocárdio. ${ }^{72}$ Estes efeitos são abolidos quando os resultados são ajustados pelos fatores de risco para aterosclerose, particularmente HDL-colesterol, demonstrando que elevados níveis de t-PA são mais um resultado do que uma causa para aterosclerose. Isso evidencia, então, que os baixos níveis de t-PA antígeno nos atletas são benéficos na redução dos riscos de doenças cardiovasculares.

\section{Conclusão}

A atividade física regular está associada à diminuição dos riscos de doenças cardiovasculares, incluindo a redução da tendência trombogênica pela diminuição da atividade coagulante e aumento da atividade fibrinolítica. Um desequilíbrio nos mecanismos envolvidos na hemostasia gera um quadro patológico resultando em sangramento excessivo ou trombose. Alguns autores sugerem adaptações promovidas pelo treinamento, principalmente no potencial fibrinolítico, que diminuem o risco de trombose arterial coronariana.

Os estudos acerca dos mecanismos pelos quais o exercício modifica o processo hemostático ainda necessitam de conclusões mais contundentes. Agudamente, o exercício aumenta as respostas coagulantes e fibrinolíticas. Já os efeitos crônicos são positivos em pessoas com deficiências nestes processos (por exemplo, pacientes pós-infarto do miocárdio). Mais estudos são necessários, principalmente quanto aos mecanismos pelos quais o exercício modifica esses processos, não só para entendê-los mas também para aplicar estes conhecimentos na prevenção e tratamento das doenças relacionadas.

\section{Abstract}

Post-exercise hypercoagulability state has been well demonstrated as increases in coagulation activity are often reported after many exercise protocols. One of the most consistent effects of acute exercise is an increase in coagulation activity reflected by the FVIII activity of thrombin generation markers (TAT and F1+2). This increase is intensity-dependent. In relation to chronic effects, resting coagulant activity does not change with training. However, post-exercise FVIII levels have been reported to be greater in sedentary individuals than in trained subjects and resting levels of post-infarction patients decrease with training. Hyperfibrinolysis related to exercise has also been reported and, similar to coagulation activity, may be intensity-dependent. Increases in $t-P A, u-P A$, and plasminogen levels and decreases in PAI-1 after exercise have been reported. Resting t-
$P A$ levels of non-trained individuals are higher than in active subjects or athletes. In addition, post-infarction patients have a significant decrease of PAI-1 levels after rehabilitation. More controversial results are related to the chronic effects of $t-P A$. While some studies have shown higher levels of t-PA in trained individuals compared with non-trained subjects, others have not shown this. More consistent results related to the effects of exercise on the hemostatic process, including coagulation and fibrinolytic activities, remain necessary. Chronic effects of exercise need to be studied further, both in relation to the health benefits and the influence on the performance of athletes. Rev. bras. hematol. hemoter. 2005;27(3):213-220.

Key words: Coagulation; fibrinolysis; hemostasy; exercise; training.

\section{Referências Bibliográficas}

1. Cannon WB. Bodily changes in pain, hunger, fear and rage; an account of recent researches into the function of emotional excitement. $2 \mathrm{~d}$ ed. New York, London: D. Appleton and company; 1929.

2. Dufaux B, Order U, Liesen H. Effect of a short maximal physical exercise on coagulation, fibrinolysis, and complement system. Int J Sports Med 1991;12 Suppl 1:S38-42.

3. Andrew M, Carter C, O'Brodovich $\mathrm{H}$ et al. Increases in factor VIII complex and fibrinolytic activity are dependent on exercise intensity. J Appl Physiol 1986;60(6):1.917-22.

4. Huisveld IA, Hospers AJ, Bernink MJ et al. Oral contraceptives and fibrinolysis among female cyclists before and after exercise. J Appl Physiol 1982;53(2):330-4.

5. Bourey RE, Santoro SA. Interactions of exercise, coagulation, platelets, and fibrinolysis - a brief review. Med Sci Sports Exerc 1988;20(5):439-46.

6. Collen D, Semeraro N, Tricot JP et al. Turnover of fibrinogen, plasminogen, and prothrombin during exercise in man. J Appl Physiol 1977;42(6):865-73.

7. Ferguson EW, Barr CF, Bernier LL. Fibrinogenolysis and fibrinolysis with strenuous exercise. J Appl Physiol 1979;47(6):1.157-61.

8. Small M, Tweddel AC, Rankin AC et al. Blood coagulation and platelet function following maximal exercise: effects of betaadrenoceptor blockade. Haemostasis 1984;14(3):262-8.

9. Vicente V, Alberca I, Mannucci PM. Reduced effect of exercise and DDAVP on factor VIII-von Willebrand factor and plasminogen activator after sequential application of both the stimuli. Thromb Haemost 1984;51(1):129-30.

10. Marsh N, Gaffney P. Some observations on the release of extrinsic and intrinsic plasminogen activators during exercise in man. Haemostasis 1980;9(4):238-47.

11. Stibbe J. Effect of exercise on F VIII-complex: proportional increase of ristocetin cofactor (Von Willebrand factor) and F VIII-AGN, but disproportional increase of F VIII-AHF. Thromb Res 1977; 10(1):163-8.

12. Wheeler ME, Davis GL, Gillespie WJ et al. Physiological changes in hemostasis associated with acute exercise. J Appl Physiol 1986; 60(3):986-90.

13. Brown JE, Baugh RF, Hougie C. Effect of exercise on the factor VIII complex: a correlation of the Von Willebrand antigen and factor VIII coagulant antigen increase. Thromb Res 1979;15(1-2):61-7.

14. Kopitsky RG, Switzer ME, Williams RS et al. The basis for the increase in factor VIII procoagulant activity during exercise. Thromb Haemost 1983;49(1):53-7. 
15. Jilma B, Dirnberger E, Eichler HG et al. Partial blockade of nitric oxide synthase blunts the exercise-induced increase of von Willebrand factor antigen and of factor VIII in man. Thromb Haemost 1997;78(4):1.268-71.

16. Cohen RJ, Epstein SE, Cohen LS et al. Alterations of fibrinolysis and blood coagulation induced by exercise, and the role of betaadrenergic-receptor stimulation. Lancet 1968;2(7581):1.264-6.

17. Ikarugi H, Taka T, Nakajima S et al. Norepinephrine, but not epinephrine, enhances platelet reactivity and coagulation after exercise in humans. J Appl Physiol 1999;86(1):133-8.

18. Wagner CT, Kroll MH, Chow TW et al. Epinephrine and shear stress synergistically induce platelet aggregation via a mechanism that partially bypasses VWF-GP IB interactions. Biorheology 1996;33(3):209-29.

19. van den Burg PJ, Hospers JE, Mosterd WL et al. Aging, physical conditioning, and exercise-induced changes in hemostatic factors and reaction products. J Appl Physiol 2000;88(5):1.558-64.

20. Rock G, Tittley P, Pipe A. Coagulation factor changes following endurance exercise. Clin J Sport Med 1997;7(2):94-9.

21. Arai M, Yorifuji H, Ikematsu S et al. Influences of strenuous exercise (triathlon) on blood coagulation and fibrinolytic system. Thromb Res 1990;57(3):465-71.

22. El-Sayed MS. Effects of high and low intensity aerobic conditioning programs on blood fibrinolysis and lipid profile. Blood Coagul Fibrinolysis 1996;7(4):484-90.

23. Hansen JB, Wilsgard L, Olsen JO et al. Formation and persistence of procoagulant and fibrinolytic activities in circulation after strenuous physical exercise. Thromb Haemost 1990;64(3):385-9.

24. El-Sayed MS. Fibrinolytic and hemostatic parameter response after resistance exercise. Med Sci Sports Exerc 1993;25(5):597-602.

25. Davis GL, Abildgaard CF, Bernauer EM et al. Fibrinolytic and hemostatic changes during and after maximal exercise in males. J Appl Physiol 1976;40(3):287-92.

26. Salton G, Ribeiro J, Bandinelli E et al. Efeitos de diferentes intensidades de exercício nos níveis plasmáticos do fator von Willebrand. Rev Bras Med Esporte 2002;8(5):203.

27. Bartsch P, Schmidt EK, Straub PW. Fibrinopeptide A after strenuous physical exercise at high altitude. J Appl Physiol 1982;53(1):40-3.

28. Hyers TM, Martin BJ, Pratt DS et al. Enhanced thrombin and plasmin activity with exercise in man. J Appl Physiol 1980;48 (5):821-5

29. Berkarda B, Akokan G, Derman U. Fibrinolytic response to physical exercise in males. Atherosclerosis 1971;13(1):85-91.

30. Ferguson EW, Guest MM. Exercise, physical conditioning, blood coagulation and fibrinolysis. Thromb Diath Haemorrh 1974; 31(1):63-71.

31. Rocker L, Gunay S, Gunga HC et al. Activation of blood platelets in response to maximal isometric exercise of the dominant arm. Int J Sports Med 2000;21(3):191-4.

32. Ferguson EW, Bernier LL, Banta GR et al. Effects of exercise and conditioning on clotting and fibrinolytic activity in men. J Appl Physiol 1987;62(4):1.416-21.

33. Rocker L, Taenzer M, Drygas WK et al. Effect of prolonged physical exercise on the fibrinolytic system. Eur J Appl Physiol Occup Physiol 1990;60(6):478-81

34. Molz AB, Heyduck B, Lill H et al. The effect of different exercise intensities on the fibrinolytic system. Eur J Appl Physiol Occup Physiol 1993;67(4):298-304.

35. Mandalaki T, Dessypris A, Louizou C et al. Marathon run I: effects on blood coagulation and fibrinolysis. Thromb Haemost 1977; 37 (3):444-50
36. Rizza CR. Effect of exercise on the level of antihaemophilic globulin in human blood. J Physiol 1961;156:128-35.

37. Herren T, Bartsch P, Haeberli A et al. Increased thrombinantithrombin III complexes after $1 \mathrm{~h}$ of physical exercise. J Appl Physiol 1992;73(6):2499-504.

38. Rankinen T, Vaisanen S, Penttila I et al. Acute dynamic exercise increases fibrinolytic activity. Thromb Haemost 1995;73(2):281-6.

39. Suzuki T, Yamauchi K, Yamada Y et al. Blood coagulability and fibrinolytic activity before and after physical training during the recovery phase of acute myocardial infarction. Clin Cardiol 1992; $15(5): 358-64$

40. Torres-Guerra E, Diez-Ewald M, Vizcaino G et al. [Effect of stress electrocardiogram on platelet function, concentration of von Willebrand factor and fibrinogen in hypertensive patients and healthy subjects]. Invest Clin 2000;41(2):105-16.

41. Bartsch P, Welsch B, Albert M et al. Balanced activation of coagulation and fibrinolysis after a 2-h triathlon. Med Sci Sports Exerc 1995;27(11):1465-70.

42.Prisco D, Paniccia R, Bandinelli B et al. Evaluation of clotting and fibrinolytic activation after protracted physical exercise. Thromb Res 1998;89(2):73-8.

43. El-Sayed MS, Sale C, Jones PG et al. Blood hemostasis in exercise and training. Med Sci Sports Exerc 2000;32(5):918-25.

44. Bartsch P, Haeberli A, Straub PW. Blood coagulation after long distance running: antithrombin III prevents fibrin formation. Thromb Haemost 1990;63(3):430-4.

45. Kvernmo HD, Osterud B. The effect of physical conditioning suggests adaptation in procoagulant and fibrinolytic potential. Thromb Res 1997;87(6):559-69.

46. El-Sayed MS, Lin X, Rattu AJ. Blood coagulation and fibrinolysis at rest and in response to maximal exercise before and after a physical conditioning programme. Blood Coagul Fibrinolysis 1995; $6(8): 747-52$

47. Streiff M, Bell WR. Exercise and hemostasis in humans. Semin Hematol 1994;31(2):155-65.

48. van den Burg PJ, Hospers JE van Vliet M et al. Effect of endurance training and seasonal fluctuation on coagulation and fibrinolysis in young sedentary men. J Appl Physiol 1997;82(2):613-20.

49. Boman K, Hellsten G, Bruce A et al. Endurance physical activity, diet and fibrinolysis. Atherosclerosis 1994;106(1):65-74.

50. Ponjee GA, Janssen GM, van Wersch JW. Prolonged endurance exercise and blood coagulation: a 9 month prospective study. Blood Coagul Fibrinolysis 1993;4(1):21-5.

51. Watts EJ. Haemostatic changes in long-distance runners and their relevance to the prevention of ischaemic heart disease. Blood Coagul Fibrinolysis 1991;2(2):221-5.

52. DeSouza CA, Jones PP, Seals DR. Physical activity status and adverse age-related differences in coagulation and fibrinolytic factors in women. Arterioscler Thromb Vasc Biol 1998;18(3):362-8.

53. Dufaux B, Order U, Hollmann W. Can physical exercise induce an effective fibrinolysis? Thromb Res 1984;36(1):37-43.

54. Kvernmo H, Olsen JO, Osterud B. Changes in blood cell response following strenuous physical exercise. Eur J Appl Physiol Occup Physiol 1992;64(4):318-22.

55. Schneider SH, Kim HC, Khachadurian AK et al. Impaired fibrinolytic response to exercise in type II diabetes: effects of exercise and physical training. Metabolism 1988;37(10):924-9.

56. Korsan-Bengtsen K, Wilhelmsen L, Tibblin G. Blood coagulation and fibrinolysis in relation to degree of physical activity during work and leisure time. A study based on a random sample of 54year-old men. Acta Med Scand 1973;193(1-2):73-7. 
57. Hansen JB, Svensson B, Zhang CL et al. Basal plasma concentration of tissue plasminogen activator (t-PA) and the adaption to strenuous exercise in familial hypercholesterolaemia (FH). Blood Coagul Fibrinolysis 1994;5(5):781-7.

58. Wosornu D, Allardyce W, Ballantyne D et al. Influence of power and aerobic exercise training on haemostatic factors after coronary artery surgery. Br Heart J 1992;68(2):181-6.

59. Schuit AJ, Schouten EG, Kluft C et al. Effect of strenuous exercise on fibrinogen and fibrinolysis in healthy elderly men and women. Thromb Haemost 1997;78(2):845-51.

60. Chicharro JL, Sanchez O, Bandres F et al. Platelet aggregability in relation to the anaerobic threshold. Thromb Res 1994; 75 (3):251-7.

61. Gleeson M, Blannin AK, Sewell DA et al. Short-term changes in the blood leucocyte and platelet count following different durations of high-intensity treadmill running. J Sports Sci 1995;13(2):115-23.

62. Wang JS, Jen CJ, Kung HC et al. Different effects of strenuous exercise and moderate exercise on platelet function in men. Circulation 1994;90(6):2.877-85.

63. De Paz JA, Lasierra J, Villa JG et al. Changes in the fibrinolytic system associated with physical conditioning. Eur J Appl Physiol Occup Physiol 1992;65(5):388-93.

64. Handa K, Terao Y, Mori T et al. Different coagulability and fibrinolytic activity during exercise depending on exercise intensities. Thromb Res 1992;66(5):613-6.

65. Gough SC, Whitworth S, Rice PJ et al. The effect of exercise and heart rate on fibrinolytic activity. Blood Coagul Fibrinolysis 1992; 3(2):179-82.

66. Szymanski LM, Pate RR, Durstine JL. Effects of maximal exercise and venous occlusion on fibrinolytic activity in physically active and inactive men. J Appl Physiol 1994;77(5):2305-10.

67. Stegnar M, Peternel P, Chen JP. Acute hypoxemia does not increase blood fibrinolytic activity in man. Thromb Res 1987; 45(4):333-43.

68. Womack CJ, Ivey FM, Gardner AW et al. Fibrinolytic response to acute exercise in patients with peripheral arterial disease. Med Sci Sports Exerc 2001;33(2):214-9.

69. De Paz JA, Villa JG, Vilades E et al. Effects of oral contraceptives on fibrinolytic response to exercise. Med Sci Sports Exerc 1995; 27(7):961-6.

70. Speiser W, Langer W, Pschaick A et al. Increased blood fibrinolytic activity after physical exercise: comparative study in individuals with different sporting activities and in patients after myocardial infarction taking part in a rehabilitation sports program. Thromb Res 1988;51(5):543-55.

71. Estelles A, Aznar J, Tormo G et al. Influence of a rehabilitation sports programme on the fibrinolytic activity of patients after myocardial infarction. Thromb Res 1989;55(2):203-12.

72. Ridker PM, Vaughan DE, Stampfer MJ et al. Endogenous tissuetype plasminogen activator and risk of myocardial infarction. Lancet 1993;341(8854):1.165-8.

Avaliação: Editor e dois revisores externos.

Conflito de interesse: não declarado

Recebido: 12/05/2005

Aceito após modificações: 06/09/2005 